\title{
Vorwort zur 1. Auflage.
}

Obgleich das Motorrad älter als das Automobil mit Verbrennungskraftmaschine, so ist trotzdem der Notorradbau in technisch-literarischer Hinsicht fast überhaupt nicht bearbeitet worden und finden sich hierüber, in Zeitschriften verstreut, nur wenige Angaben von außerdem recht zweifelhaftem Wert. Die bestehenden Arbeiten, die ausschließlich den Motorradbau behandeln, gestatten kaum einen Allgemeinüberblick über diesen Industriezweig, geschweige denn eine tiefgreifende Information. Es war somit technischen Interessenten, besonders Konstrukteuren aus anderen verwandten Fachgebieten sowie Studierenden unmöglich, sich in diese Materie einzuarbeiten. Durch vorliegende Arbeit war ich bestrebt, diese Lücke auszufüllen.

Die Bearbeitung dieses Stoffes machte außerordentlich große Mülne, da sich, von wenigen rühmlichen Ausnahmen abgesehen, die in Betracht kommende Industrie jeder Auskunft enthielt, die nur irgendwelchen Wert für die Ausnutzung und Weiterbearbeitung haben könnte. Wenn dieser Standpunkt auch in gewisser Beziehung zu verstehen ist, so wird der Sache selbst mit diesem Verhalten jedoch sicherlich nicht gedient. Wissenschaftliche Untersuchungen sind, soweit sie lediglich den Motorradbau betreffen, nur in verschwindend wenig Fällen durchgeführt worden, so daß konstruktive Arbeit nur unter Berücksichtigung empirischer Werte geleistet wurde.

Durch diese Ausarbeitung ist versucht worden, den heutigen Stand der gesamten Motorradindustrie festzulegen, die konstruktiven Gesichtspunkte einer zusammenhängenden Darstellung und Kritik zu unterziehen sowie Anregungen zur Behebung von Fehlern zu geben.

In Anbetracht dieses äußerst umfangreichen und vielseitigen Spezialfaches ist es sehr wohl-möglich, daß mir dieses oder jenes bei der Behandlung des Stoffes entgangen ist. Ich möchte daher die Herrn Kollegen bitten, mir für die Ausarbeitung sowie Ergänzung der nächsten Auflage mit diesbezüglichen Hinweisen und Unterlagen an die Hand zu gehen, wofür ich im voraus meinen verbindlichsten Dank ausspreche.

Berlin, im Oktober 1919. 
